\title{
Propriedades termo-mecânicas de concretos refratários ligados por sílica coloidal
}

\section{(Thermo-mechanical properties of colloidal silica-containing castable)}

\author{
M. R. Ismael, F. A. O. Valenzuela, L. A. Polito, V. C. Pandolfelli \\ Departamento de Engenharia de Materiais - Universidade Federal de S.Carlos \\ Rod. Washington Luiz, km 235, C.P. 676, S. Carlos, SP 13565-905 \\ marinaismael@polvo.ufscar.br,vicpando@power.ufscar.br
}

\begin{abstract}
Resumo
Os cimentos de aluminatos de cálcio (CAC) são os ligantes hidráulicos mais utilizados em concretos refratários. No entanto, a presença de $\mathrm{CaO}$ em sua composição pode limitar seu uso em elevadas temperaturas nos sistemas $\mathrm{Al}_{2} \mathrm{O}_{3}-\mathrm{SiO}_{2}$ e $\mathrm{Al}_{2} \mathrm{O}_{3}-\mathrm{MgO}$. Como importante alternativa ao uso do CAC, destaca-se a sílica coloidal, que proporciona, dentre outras vantagens, maior velocidade de secagem e reatividade ao sistema. Estudos anteriores dos autores deste trabalho indicaram que a adição de microssílica e alumina hidratável podem otimizar o desempenho de concretos contendo sílica coloidal como agente ligante. No entanto, investigações sobre esses sistemas em elevadas temperaturas de trabalho são escassas na literatura. Neste trabalho, o comportamento em diferentes temperaturas de sistemas contendo sílica coloidal foi avaliado quanto à retração linear após queima, porosidade aparente, resistência mecânica a frio e a quente. Como referência, foi utilizada uma formulação de concreto contendo cimento de aluminato de cálcio como agente ligante.
\end{abstract}

Palavras-chave: concretos refratários, agentes ligantes, sílica coloidal, propriedades termo-mecânicas.

\begin{abstract}
Calcium aluminate cements $(C A C)$ are the most used hydraulic binders in refractory castables. However the presence of CaO in the castable composition reduces the refractoriness in the $\mathrm{Al}_{2} \mathrm{O}_{3}-\mathrm{SiO}_{2}$ and $\mathrm{Al}_{2} \mathrm{O}_{3}-\mathrm{MgO}$ systems. In this context, colloidal silica has been pointed out as an important alternative for the CAC use because promotes, among another advantages, easier drying behavior and higher reactivity to the system. Previous works of the authors have indicated that the microsilica and hydratable alumina addition to colloidal silica containing castables can optimize their performance. Nevertheless, researches in these systems at high working temperatures (above $350{ }^{\circ} \mathrm{C}$ ) are scarce in the literature. In this work, the colloidal silica bonded castables behaviors at different working temperature were analyzed through the fired linear shrinkage, apparent porosity, mechanical strength and hot modulus of rupture. The obtained results were compared to the CAC containing castable compositions.
\end{abstract}

Keywords: refractory castables, binder agents, colloidal silica, thermo-mechanical properties.

\section{INTRODUÇ̃̃O}

O principal agente ligante para concretos refratários é o cimento de aluminato de cálcio (CAC), principalmente por promover elevada resistência mecânica a verde e resistência a agentes corrosivos [1]. No entanto, a presença de cálcio na sua composição pode limitar seu uso em elevadas temperaturas nos sistemas $\mathrm{Al}_{2} \mathrm{O}_{3}-\mathrm{SiO}_{2}$ e $\mathrm{Al}_{2} \mathrm{O}_{3}-\mathrm{MgO}$, devido a formação de fases de baixo ponto de fusão. Nesse contexto, tem sido sugerido como importante alternativa a substituição do CAC pela sílica coloidal (SC) [2-8].

A sílica coloidal é uma dispersão estável de partículas nanométricas de sílica amorfa, que por meio da gelificação do sol (ligação silano: -Si-O-Si-), desenvolve resistência mecânica pela formação de uma rede tridimensional entre as partículas [9]. Sílica coloidal como agente ligante para concretos refratários proporciona maior facilidade de mistura, além de ser menos susceptível às condições de cura que o CAC tradicionalmente empregado [2-8]. Além disso, devido ao mecanismo de consolidação do sol, a secagem desses sistemas é extremamente rápida, apresentando pouco risco de explosão durante esta etapa do processo. Quando aplicada em concretos refratários aluminosos, a elevada área superficial das partículas de sílica coloidal (em torno de 220 $\mathrm{m}^{2} / \mathrm{g}$ ) aumenta a reatividade do sistema, podendo gerar mulita in-situ, dando origem a composições com maior resistência ao ataque químico e resistência mecânica a quente [6-8].

Estudos anteriores dos autores deste trabalho mostraram o efeito da adição de microssílica e alumina hidratável em sistemas ligados por sílica coloidal $[8,10]$. A presença de microssílica, além de aumentar a resistência mecânica do sistema, favorece a sinterabilidade, proporcionando menor fluência a quente [8]. A combinação de sílica coloidal e alumina hidratável gera sistemas com reduzida porosidade, aliada a elevados valores de resistência mecânica a verde (abaixo de $350{ }^{\circ} \mathrm{C}$ ) [10]. No entanto, investigações sobre 
esses sistemas em superiores temperaturas de trabalho (acima de $350{ }^{\circ} \mathrm{C}$ ) são escassas na literatura. Deste modo, o objetivo deste trabalho foi avaliar as propriedades dos concretos contendo sílica coloidal como agente ligante após tratamento térmico em diferentes temperaturas. A retração linear após queima, porosidade aparente e a resistência mecânica a frio e a quente foram comparadas as propriedades de um concreto ligado por CAC.

\section{MATERIAIS E MÉTODOS}

A formulação das composições exploradas foi realizada baseando-se no modelo de empacotamento de partículas de Andreasen [11], com coeficiente (q) 0,21 e diâmetro máximo de partícula igual a $4750 \mu \mathrm{m}$. As matérias-primas utilizadas na formulação de cada sistema são apresentadas na Tabela I.

Após mistura das matérias-primas em um reômetro desenvolvido para concretos [12], as diferentes composições foram moldadas em barras metálicas de $25 \times 25 \times 125 \mathrm{~mm}$. A cura foi realizada a $50^{\circ} \mathrm{C}$ durante $24 \mathrm{~h}$ em câmara climatizada (Vötsch 2020). As composições contendo sílica coloidal (SC, MS e ABS) foram curadas em ambiente insaturado (ao ar), enquanto que a composição CAC foi curada em ambiente saturado de vapor de água [6]. Os tratamentos térmicos foram realizados com uma taxa de aquecimento de $3{ }^{\circ} \mathrm{C} /$ min, com patamar de $12 \mathrm{~h}$ nas seguintes temperaturas: 110 , $250,500,750,1000,1250$ e $1500{ }^{\circ} \mathrm{C}$. A taxa de resfriamento utilizada foi de $10^{\circ} \mathrm{C} / \mathrm{min}$.

A medida de retração linear após queima (RLQ - equação A) foi feita com a média de 3 comprimentos iniciais ( $L_{0}$ - antes da queima) de cada corpo de prova e 3 comprimentos finais $\left(\mathrm{L}_{\mathrm{f}}\right.$ - antes da queima). A retração linear de cada temperatura para cada sistema estudado equivale a média de 5 corpos de provas.

$$
\operatorname{RLQ}(\%)=\left(\frac{\mathrm{L}_{\mathrm{f}}-\mathrm{L}_{\mathrm{o}}}{\mathrm{L}_{\mathrm{o}}}\right) \times 100
$$

onde $\mathrm{L}_{\mathrm{f}}=$ comprimento final e $\mathrm{L}_{\mathrm{o}}=$ comprimento inicial.

A porosidade aparente dos concretos foi obtida pelo método de imersão de Archimedes, utilizando querosene como líquido de imersão (norma ASTM C 830).

Para o ensaio de resistência mecânica (flexão a 3 pontos - norma ASTM C133-97, 2003) foi utilizada a máquina de ensaios universal MTS 810. A tensão de ruptura foi calculada a partir da equação B. Para este ensaio, os corpos de provas previamente tratados termicamente foram retificados a úmido (retifica Fagor Automation, Modelo Nv 20) e posteriormente secos a $110^{\circ} \mathrm{C}$ por, no mínimo, durante $24 \mathrm{~h}$. Foram ensaiados 5 corpos de provas para cada composição.

$$
\sigma_{\mathrm{r}}=\frac{3}{2} \cdot \frac{\mathrm{P}_{\max } \mathrm{L}}{\mathrm{b} \cdot \mathrm{h}^{2}}
$$

onde $\mathrm{P}_{\max }$ é a força de ruptura, $\mathrm{L}$ a distância entre os apoios (125 mm) e b e h são a largura e a altura do corpo-de-prova, respectivamente.

Para a determinação do módulo de ruptura a quente (M.R.Q.) foi utilizada uma máquina de flexão a três pontos, modelo HBST (High Bending Strength Tester) 422, marca Netzsch. A velocidade de carregamento foi de $12,5 \mathrm{~N} / \mathrm{s}$, de acordo com a norma ASTM C 583-8. O cálculo do módulo de ruptura $\sigma_{\mathrm{r}}$ foi feito segundo a equação $\mathrm{B}$. As amostras foram inicialmente sinterizadas nas respectivas temperaturas de ensaio por $12 \mathrm{~h}\left(1150,1300\right.$ e $1450{ }^{\circ} \mathrm{C}$ taxa de aquecimento $3{ }^{\circ} \mathrm{C} / \mathrm{min}$ ) e posteriormente retificadas

Tabela I - Matérias-primas utilizadas na composição dos concretos.

\begin{tabular}{|c|c|c|c|c|}
\hline \multirow{2}{*}{$\begin{array}{c}\text { Matérias-Primas } \\
\text { (Especificações - Fornecedor) }\end{array}$} & \multicolumn{3}{|c|}{ Composições } & \multirow[b]{2}{*}{ CAC } \\
\hline & $\mathrm{SC}$ & MS & ABS & \\
\hline $\begin{array}{l}\text { Alumina Calcinada (\%-p) } \\
\text { (E-SY PUMP } 1000 \text { - Almatis) }\end{array}$ & 34,0 & 31,0 & 30,0 & 33,0 \\
\hline $\begin{array}{l}\text { Microssílica (\%-p) } \\
\text { (971-D - Elkem) }\end{array}$ & - & 3,0 & - & - \\
\hline $\begin{array}{l}\text { Alumina Eletrofundida Marrom (\%-p) } \\
\text { (Elfusa - Brasil) }\end{array}$ & 66,0 & 66,0 & 70,0 & 67,0 \\
\hline $\begin{array}{c}\text { Ligante (\%-p) } \\
\text { CAC (CA-14M - Almatis), SC (Sílica Coloidal Nalco), } \\
\text { AB (Alphabond } 300 \text { - Almatis) }\end{array}$ & 7,5 SC & $7,5 \mathrm{SC}$ & $\begin{array}{l}5,0 \mathrm{SC} \mathrm{e} \\
3,0 \mathrm{AB}\end{array}$ & $3,0 \mathrm{CAC}$ \\
\hline $\begin{array}{l}\text { Teor de } \mathrm{H}_{2} \mathrm{O}(\%-\mathrm{p}) \\
\text { Dispersante }\left(\mathrm{mg} / \mathrm{m}^{2}\right)\end{array}$ & 4,5 & 4,5 & 4,5 & 4,5 \\
\hline $\begin{array}{c}\left(\mathrm{PEG}^{*} \text { ou Ácido Cítrico }(\mathrm{AC})\right) \\
\text { Gelificante }(\%-\mathrm{p})^{* *}\end{array}$ & $0,36 \mathrm{AC}$ & 0,78 PEG & $0,36 \mathrm{AC}$ & $0,36 \mathrm{AC}$ \\
\hline (Sínter de $\mathrm{MgO}$ ) & 0,6 & 0,6 & 0,6 & - \\
\hline
\end{tabular}

[Table I - Castables formulation and raw material.]

*PEG $=$ Polietilenoglicol, $* * \%$ - $p$ em relação à quantidade de sílica coloidal. 
a úmido. Foram utilizados 5 corpos de provas para cada temperatura de ensaio.

\section{RESULTADOS E DISCUSSÃO}

A Fig. 1 apresenta os valores de retração linear para as composições analisadas em diferentes temperaturas. A retração linear após queima relaciona-se, de forma geral, com as fases formadas nos concretos. A $110{ }^{\circ} \mathrm{C}$ nenhum corpo de prova apresentou retração. O sistema contendo somente a sílica coloidal (SC) apresentou início de retração apenas a partir de $500{ }^{\circ} \mathrm{C}$. Menores valores de retração nas temperaturas de 1250 e $1500{ }^{\circ} \mathrm{C}$ eram esperados para os sistemas contendo sílica coloidal, devido a provável formação da fase mulita, geralmente acompanhada de expansão. Este aspecto é comprovado pela composição MS, que devido ao maior teor total de sílica $(6 \%$ - peso, Tabela I), retraiu menos, provavelmente pelo maior teor de mulita.

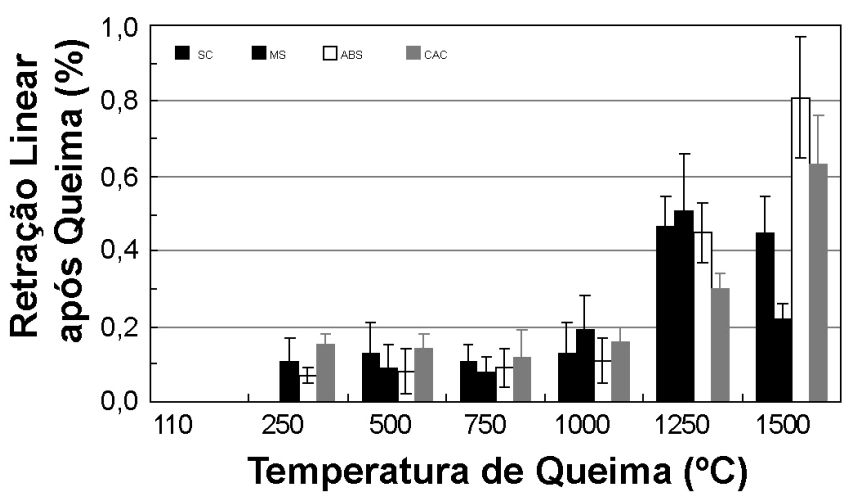

Figura 1: Retração linear após queima para as composições contendo sílica coloidal (SC), sílica coloidal e microssílica (MS), sílica coloidal e alumina hidratável (ABS) e cimento de aluminato de cálcio (CAC).

[Figure 1: Fired linear shrinkage for the compositions containing colloidal silica (SC), colloidal silica and microsilica (MS), colloidal silica and hydratable alumina $(A B S)$ and calcium aluminate cement (CAC).]

A porosidade aparente e a resistência mecânica em função da temperatura de queima são apresentadas nas Figs. 2 e 3, respectivamente. A diminuição da porosidade nas temperaturas de 1250 e $1500{ }^{\circ} \mathrm{C}$ para os sistemas SC, MS e ABS foi acompanhada por uma maior retração linear. Essa redução na porosidade pode ser atribuída a provável formação de um líquido meta-estável silico-aluminoso em aproximadamente $1260^{\circ} \mathrm{C}$ [13], favorecendo a sinterização e o fechamento dos poros.

O fato de que a saída da água estrutural deixa vazios é evidenciado para as composições que formam fases hidratadas (ABS e CAC), nas quais a porosidade inicial $\left(110{ }^{\circ} \mathrm{C}\right)$ foi em torno de 10 e $9 \%$, e na temperatura de $750{ }^{\circ} \mathrm{C}$, foi de 14 e $12 \%$, respectivamente. Consequentemente, na Fig. 3 verifica-se uma certa queda de resistência mecânica em temperaturas até $750^{\circ} \mathrm{C}$ para esses sistemas, uma vez que ocorre a decomposição das

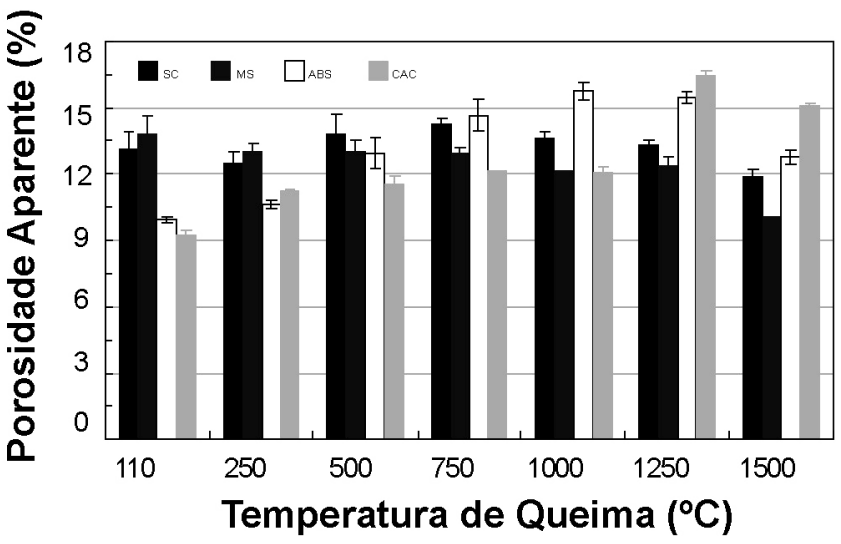

Figura 2: Porosidade aparente em função da temperatura de queima para as composições contendo sílica coloidal (SC), sílica coloidal e microssílica (MS), sílica coloidal e alumina hidratável (ABS) e cimento de aluminato de cálcio (CAC).

[Figure 2: Apparent porosity as a function of curing temperature for the compositions containing colloidal silica (SC), colloidal silica and microsilica (MS), colloidal silica and hydratable alumina $(A B S)$ and calcium aluminate cement (CAC).]

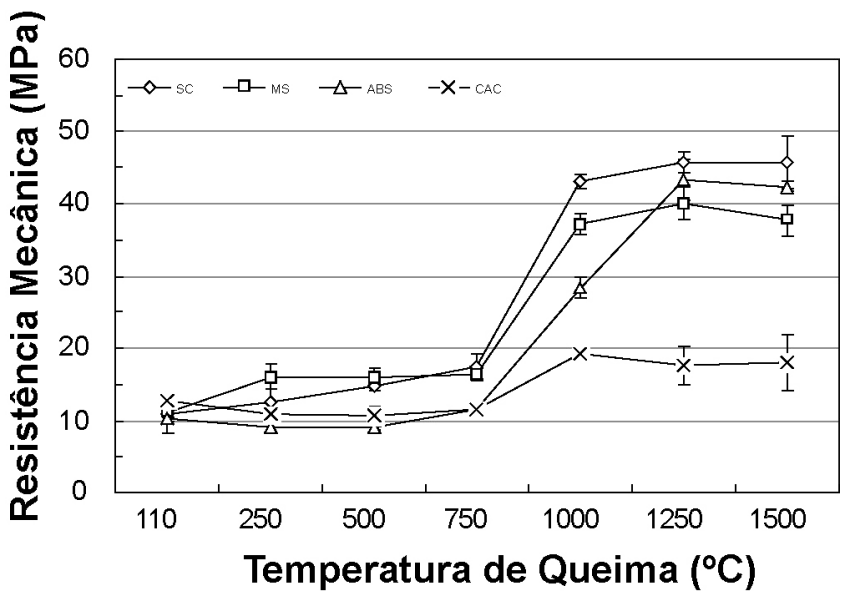

Figura 3: Resistência mecânica a frio em função da temperatura de queima para as composições contendo sílica coloidal (SC), sílica coloidal e microssílica (MS), sílica coloidal e alumina hidratável (ABS) e cimento de aluminato de cálcio (CAC).

[Figure 3: Cold mechanical strength as a function of fired temperature for the compositions containing colloidal silica (SC), colloidal silica and microsilica (MS), colloidal silica and hydratable alumina $(A B S)$ and calcium aluminate cement (CAC).]

fases e geração de poros. A partir de $1000{ }^{\circ} \mathrm{C}$, um aumento considerável na resistência mecânica desses sistemas é observado, o que pode ser atribuído à ligação cerâmica. Já para os sistemas SC e MS, ambos contendo apenas sílica coloidal, como não há a formação de complexos com a água, a resistência mecânica apresentou-se superior aos sistemas ABS e CAC entre 250 e $750{ }^{\circ} \mathrm{C}$.

Apesar da baixa resistência mecânica inicial constatada em trabalhos anteriores (imediatamente após a cura a $50^{\circ} \mathrm{C}$ ) para sistemas ligados com sílica coloidal [6], essas composições apresentaram um considerável progresso no desenvolvimento 
de resistência mecânica com a temperatura $\left(750-1500{ }^{\circ} \mathrm{C}\right)$. Tal fato pode estar associado a grande reatividade da sílica coloidal e a formação de mulita.

Os resultados obtidos quanto à caracterização do módulo de ruptura a quente desses sistemas são indicados na Fig. 4. O ensaio de M.R.Q. em elevadas temperaturas simula a condição mais próxima de trabalho do concreto.

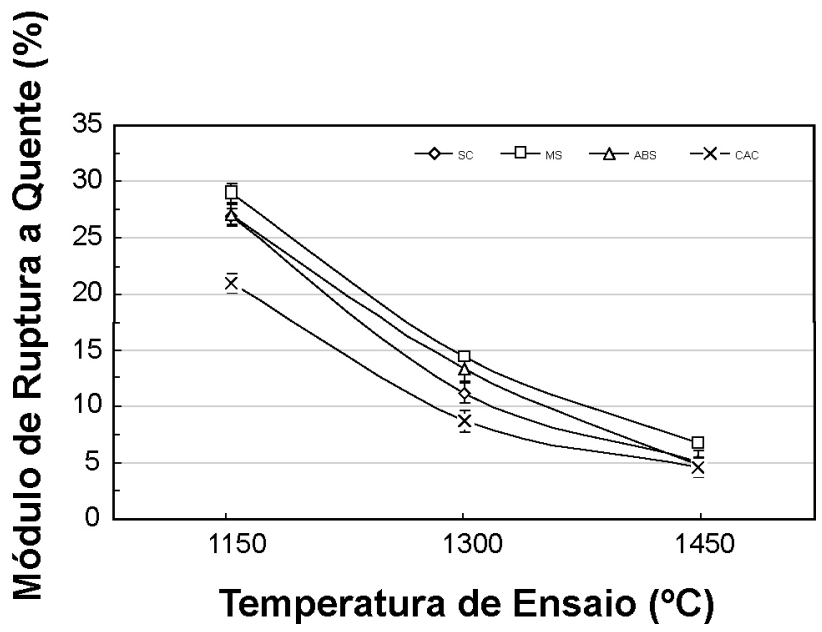

Figura 4: Módulo de ruptura a quente (MPa) em função da temperatura de ensaio para as composições contendo sílica coloidal (SC), sílica coloidal e microssílica (MS), sílica coloidal e alumina hidratável (ABS) e cimento de aluminato de cálcio (CAC).

[Figure 4: Hot modulus of rupture (MPa) as a function of temperature for the compositions containing colloidal silica (SC), colloidal silica and microsilica (MS), colloidal silica and hydratable alumina $(A B S)$ and calcium aluminate cement (CAC).]

$\mathrm{Na}$ temperatura de $1150{ }^{\circ} \mathrm{C}$ as formulações contendo sílica coloidal apresentaram valores de resistência mecânica na faixa de 25 a $30 \mathrm{MPa}$. Dentre essas composições, a que contém microssílica (MS) apresentou o maior valor de resistência mecânica, o que pode estar associado ao menor valor de porosidade encontrado (Fig. 5). A microssílica tem forma e tamanho favoráveis para promover um superior empacotamento do concreto [8]. De forma geral, o empacotamento granulométrico projetado para as partículas da matriz dos sistemas investigados, proporcionou uma adequada resistência mecânica a $1150^{\circ} \mathrm{C}$.

A $1300{ }^{\circ} \mathrm{C}$ a resistência mecânica foi reduzida consideravelmente para os concretos contendo sílica coloidal, atingindo valores próximos a $14 \mathrm{MPa}$. Tal fato, já verificado na literatura para concretos aluminosos contendo fontes de sílica [13], pode estar associado à formação de uma fase metaestável.

O trabalho de Risbud e Pask [14] sobre o diagrama $\mathrm{Al}_{2} \mathrm{O}_{3}-\mathrm{SiO}_{2}$ mostra que mesmo utilizando matériasprimas de alta pureza pode-se obter a formação de uma fase metaestável de baixa viscosidade em temperaturas próximas a $1260^{\circ} \mathrm{C}$ (Fig. 6). De acordo com esse diagrama, verifica-se que para ocorrer a formação dessa fase, a porção reativa dos concretos (partículas $<100 \mu \mathrm{m}$ ) deveria ter

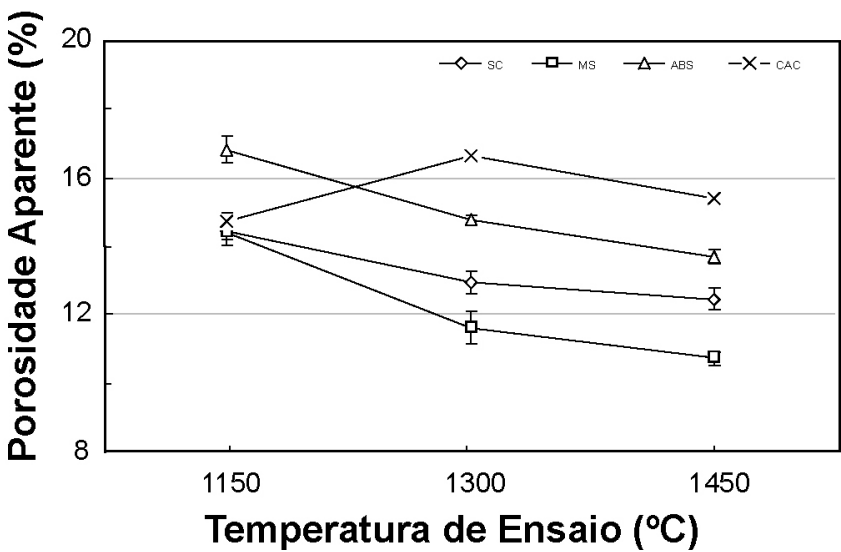

Figura 5: Porosidade Aparente (\%) em função da temperatura de ensaio para as composições contendo sílica coloidal (SC), sílica coloidal e microssílica (MS), sílica coloidal e alumina hidratável (ABS) e cimento de aluminato de cálcio (CAC).

[Figure 5: Apparent porosity (\%) as a function of temperature for the compositions containing colloidal silica (SC), colloidal silica and microsilica (MS), colloidal silica and hydratable alumina $(A B S)$ and calcium aluminate cement (CAC).J

sua estequiometria na região A indicada no diagrama. No entanto, segundo a Tabela II, a estequiometria da matriz das composições contendo sílica coloidal analisadas estaria mais próxima da região $\mathrm{B}$, onde não se verifica a formação de eutéticos. Uma provável explicação seria o fato da sílica coloidal, apesar de nanométrica, formar aglomerados durante a gelificação. Próximo a esses aglomerados, a concentração de sílica em relação a alumina aumenta, o que geraria pontualmente uma composição na região $\mathrm{A}$, favorecendo a formação de líquidos. Investigações adicionais que possibilitem esclarecer este aspecto são necessárias.

A $1450{ }^{\circ} \mathrm{C}$, a resistência mecânica dos sistemas em estudo diminuiu consideravelmente para valores próximos a $5 \mathrm{MPa}$. Nessa temperatura, para as composições com sílica coloidal, esperava-se um maior valor do módulo de

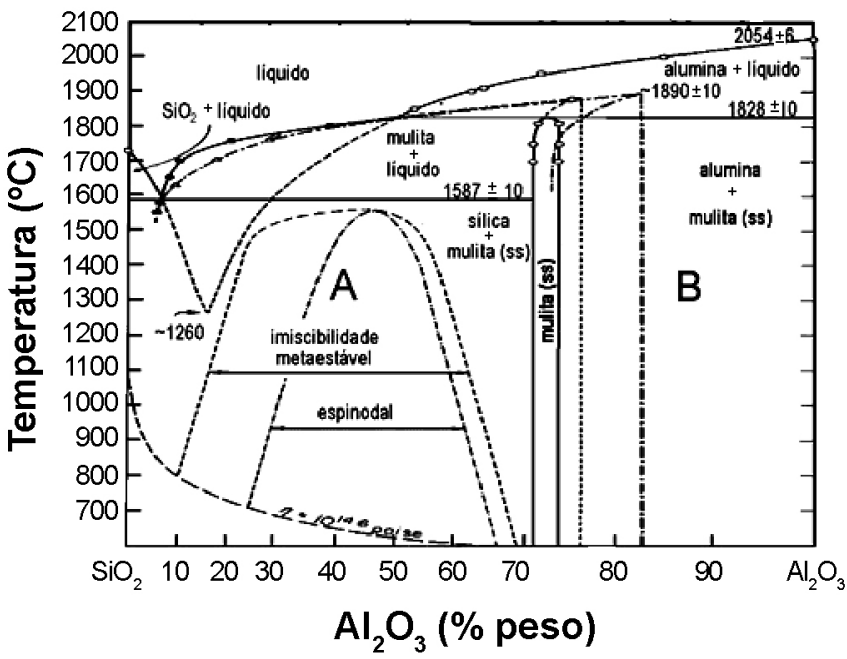

Figura 6: Diagrama $\mathrm{Al}_{2} \mathrm{O}_{3}-\mathrm{SiO}_{2}$ proposto por Risbud e Pask [14]. [Figure 6: $\mathrm{Al}_{2} \mathrm{O}_{3}-\mathrm{SiO}_{2}$ binary system by Risbud and Pask [14].] 
Tabela II - Relação em peso $\mathrm{Al}_{2} \mathrm{O}_{3}: \mathrm{SiO}_{2}$ da matriz das composições contendo sílica coloidal.

[Table II - $\mathrm{Al}_{2} \mathrm{O}_{3}: \mathrm{SiO}_{2}$ ratio in the matrix of colloidal silica containing castables.]

\begin{tabular}{lccr}
\hline Composições & $\mathrm{SC}$ & $\mathrm{MS}$ & ABS \\
\hline $\mathrm{Al}_{2} \mathrm{O}_{3}: \mathrm{SiO}_{2}$ & $92,0: 8,0$ & $84,0: 16,0$ & $94,0: 6,0$ \\
\hline
\end{tabular}

ruptura a quente, devido a presença de mulita. No entanto, a possível aglomeração durante a gelificação pode ter inibido o aumento de resistência mecânica. Adicionalmente, como a mulita precipita a partir do líquido silicoso, o uso de pequenas quantidades de sílica $(<5 \%$-p) favorece a formação do líquido com pouca precipitação de mulita, resultando em concretos com propriedades à quente inferiores. Além disso, igualmente para o sistema $\mathrm{CAC}$, a redução da resistência mecânica com o aumento da temperatura pode estar associada à presença de impurezas como o sódio, normalmente encontradas em aluminas calcinadas, o que reduz a refratariedade do sistema.

Analisando-se a porosidade aparente dessas composições (Fig. 5), verifica-se que houve considerável aumento na densificação dos corpos contendo sílica coloidal, indicativo de alta sinterabilidade. No entanto, tal fato não contribuiu para o aumento da resistência mecânica a quente. Isto pode estar associado à formação de líquido, segundo a hipótese formulada (região $\mathrm{A}$ do diagrama $\mathrm{Al}_{2} \mathrm{O}_{3}-\mathrm{SiO}_{2}$ ) ou pela quantidade de mulita formada. Os maiores valores de porosidade para as composições $\mathrm{CAC}$, refletem nos inferiores valores de M.R.Q. alcançados para esses sistemas nas condições de processamento e formulação usada neste trabalho.

\section{CONCLUSÕES}

Os resultados obtidos mostraram que na faixa de temperatura $250-750{ }^{\circ} \mathrm{C}$ as composições contendo SC, por não formarem complexos com a água, apresentaram superiores valores de resistência mecânica quando comparadas às contendo CAC. Além disso, esses sistemas apresentaram um importante progresso no desenvolvimento de resistência mecânica com a temperatura $\left(750-1500^{\circ} \mathrm{C}\right)$, o que pode estar associado a provável formação de mulita.

O módulo de ruptura a quente desses materiais indicou que o empacotamento granulométrico projetado para as partículas da matriz dos sistemas estudados proporcionou adequados valores de resistência mecânica a $1150{ }^{\circ} \mathrm{C}$. A $1300^{\circ} \mathrm{C}$, a formação de uma fase meta-estável no sistema $\mathrm{Al}_{2} \mathrm{O}_{3}-\mathrm{SiO}_{2}$, pode ter influenciado tal propriedade. A 1450 ${ }^{\circ} \mathrm{C}$, a redução de resistência mecânica observada tanto para as composições contendo SC como para as ligadas por CAC, pode ser associada à presença de impurezas como o sódio, normalmente encontradas em aluminas calcinadas.

Assim, de acordo com as propriedades verificadas quanto às análises em diferentes temperaturas de trabalho de concretos refratários ligados por sílica coloidal, é razoável considerar esses sistemas como importantes materiais para aplicações refratárias. $\mathrm{O}$ aumento de resistência mecânica a quente poderia ser obtido avaliando-se o efeito de maiores teores deste agente ligante ou utilizando-se suspensões com maiores concentrações de sólidos.

\section{AGRADECIMENTOS}

A CAPES, FAPESP e Magnesita S.A. pelo fomento à pesquisa e a Almatis e Elfusa Geral de Eletrofusão Ltda. pelas matérias-primas fornecidas.

\section{REFERÊNCIAS}

[1] K. M. Parker, J. H. Sharp, Refractory Calcium Aluminate Cements. Trans. J. Brit. Ceram. Soc. 81, 2 (1982) 35.

[2] S. Banerjee, Monolithic Refractories - a Comprehensive Handbook, World Scientific, The Am. Ceram. Soc., Singapore (1988) 111.

[3] S. Banerjee, Recent developments in monolithic refractories, Am. Ceram. Soc. Bull. 77, 10 (1998) 59.

[4] S. Banerjee, Versatility of gel-bond castable/pumpable refractories, Refract. Appl. News 6, 1 (2001) 1.

[5] S. Banerjee et al., Composition and method for manufacturing steel-containment equipment, Pat. 5.147.830 (Sept. 15, 1992).

[6] M. R. Ismael, R. D. Anjos, R. Salomão, V. C. Pandolfelli, Colloidal silica as a nanostructured binder for refractory castables, Refract. Appl. News (2006) aceito.

[7] M. R. Ismael, F. T. Ramal JR, V. C. Pandolfelli, Sol de sílica como agente ligante para concretos refratários, Cerâmica 52, 321 (2005) 84-89.

[8] M. R. Ismael, R. Salomão, V. C. Pandolfelli, Otimização do uso de sol de sílica como agente ligante para concretos refratários, Cerâmica 52, 321 (2005) 94-99.

[9] R. K. Iler, The chemistry of silica: solubility, polymerization, colloid and surface properties, and biochemistry, Wiley, New York (1979).

[10] M. R. Ismael, R. Salomão, L. A. Polito, V. C. Pandolfelli, Agentes ligantes para concretos refratários: sílica coloidal e alumina hidratável, Anais $50^{\circ} \mathrm{CBC}$ (2006).

[11] I. R. Oliveira, A. R. Studart, R. G. Pileggi, V. C. Pandolfelli, Dispersão e empacotamento de partículas - princípios e aplicações em processamento cerâmico, Fazendo Arte Editorial, S. Paulo, Brasil (2000) 119.

[12] R. G. Pileggi, V. C. Pandolfelli, A. E. Paiva, J. Gallo, Novel rheometer for refractory castables, Am. Ceram. Soc. Bull. 79, 1 (2000) 54.

[13] M. V. Gerotto, R. G. Pileggi, V.C. Pandolfelli, Resistência mecânica a quente de concretos refratários aluminosos zerocimento auto-escoantes contendo adições de microssílica e sílica coloidal, Cerâmica 46, 298 (2000) 91.

[14] S. H. Risbud, J. A. Pask, $\mathrm{SiO}_{2}-\mathrm{Al}_{2} \mathrm{O}_{3}$ metastable phase equilibrium diagram without mullite, J. Mater. Sci. 13 (1978) 2449.

(Rec. 29/04/2006, Rev. 14/08/2006, Ac. 15/12/2006) 